\title{
Office cake culture: an exploration of its characteristics and associated behaviours and attitudes among UK office workers and implications for workplace health
}

\author{
L. Walker and O. Flannery \\ Department of Clinical Sciences and Nutrition, University of Chester, Parkgate Road, Chester CH1 4 BJ.
}

The workplace is an important setting for the promotion of healthy lifestyle choices ${ }^{(1)}$. Eating frequency and energy intake from snacking have risen in recent decades ${ }^{(2)}$ with cakes and similar sweet baked goods the primary energy contributors to snack food $^{(3)}$. Providing such foods for colleagues to share, 'office cake' (OC), is a prominent form of workplace snacking and is speculated to lead to increased energy intake and obesity ${ }^{(4)}$. However no data are available. The present study explored the characteristics of OC consumption and associated attitudes and behaviours to gain insight to its impact on workplace health. OC was defined as cakes and sweet foods supplied by employees or managers to share with colleagues.

A specifically-developed 38-item cross-sectional online questionnaire based on the Theory of Planned Behaviour ${ }^{(5)}$ was completed by UK office workers in May 2017. Recruitment was through snowball and cluster sampling. Data were analysed using descriptive statistics and cross-tabulations with chi-square tests to test for between-group differences. Significance was set at $p \leq 0.05$.

Nine hundred and forty office workers (39.3\% male) participated. OC was available to $86.4 \%$ respondents between one and five times/week, $66.5 \%$ ate it at least once/week and 36.0\% never refused it. For $70.9 \%$, cake was displayed in the main working area and $58.1 \%$ said they would be less likely to eat OC if it was out of view. Attitudes and behaviour were significantly affected by gender and age. If OC is available, $41.5 \%$ said they often or always eat it, of whom significantly more were men $(48.9 \%)$ than women $(36.6 \%)$ and $18-29$ year olds $(18-29 \mathrm{~s})(52.3 \%)$ than $\geq 50$ year olds $(\geq 50 \mathrm{~s})(35.4 \%)$. Overall, women were more concerned than men about the presence and negative consequences of OC. Significantly more women than men strongly agreed/agreed that OC had contributed to weight gain (36.6\% and $23.4 \%$ respectively), made it harder to eat healthily at work $(41.4 \%$ and $31.5 \%$ respectively) and often/ always felt regret after eating it $(27.3 \%$ and $14.1 \%$ respectively). Fewer older respondents than younger approved of OC. Significantly fewer $\geq 50$ s than $18-29$ s strongly agreed/agreed OC is 'a good thing' $(53.5 \%$ and $70.9 \%$ respectively) or a good way to show appreciation $(59.7 \%$ and $82.0 \%$ respectively). Over half $(58.2 \%)$ the respondents found it hard at least sometimes to refuse OC if everyone else is eating it, with significantly more women $(25.0 \%)$ than men $(19.3 \%)$ and $18-29 \mathrm{~s}(30.2 \%)$ than $\geq 50 \mathrm{~s}(17.3 \%)$ responding 'often/always'. Almost all $(94.8 \%)$ respondents considered ideal OC frequency to be once/week or less. The mode ideal frequency was once/month $(41.3 \%)$.

OC appears to influence the UK office physical eating environment and employee dietary behaviour through increased salience and availability of energy-dense sweet foods and the effects of social influencing. Near consensus on ideal OC frequency suggests that nudge techniques could reduce OC consumption while retaining commensality benefits. These findings have implications for workplace health promotion but more research is needed.

1. Black C (2008) Working for a healthier tomorrow. London: TSO.

2. Kant AK, Graubard BI (2015) 40-year trends in meal and snack eating behaviors of American adults. J Acad Nutr Diet 115, 50-63.

3. Myhre JB, Loken EB, Wandel M et al. (2015) The contribution of snacks to dietary intake and their association with eating location among Norwegian adults - results from a cross-sectional dietary survey. BMC Public Health 15, 369.

4. Royal College of Surgeons (2017) Resolve to cut 'cake culture' in your workplace in $2017 \mathrm{https}: / /$ www.rcseng.ac.uk/news-and-events/media-centre/ press-releases/cake-culture/ (accessed June 2018)

5. Ajzen I (1991) The Theory of Planned Behavior. Organ Behav Hum Decis Process 50, 179-211. 